\title{
Epidemiology of Postpartum Depression in Pakistan: A Review of Literature
}

\author{
Razia Aliani ${ }^{*}$ and Bakhtawar Khuwaja
}

Aga Khan University Hospital, Karachi, Pakistan.

\begin{abstract}
Objective: This literature review aims to explore the risk factors, preventive measures and treatment options for women with postpartum depression especially in Pakistan.

Introduction: Postpartum depression affects $12.5 \%$ women on average and is one of the most common complications of pregnancy. Among Asian countries, Pakistan has the highest prevalence rate that is $28 \%-63 \%$. The consequences of PPD go beyond the mother and affect the partner and the child as well and can even lead to infanticide and maternal death, often by suicide. Besides, not all women are assessed for PPD or receive its treatment despite of several treatment options available.
\end{abstract}

Method: Comprehensive and systematic literature search was undertaken by using various electronic research databases. Studies were eligible for this review if they were published in last twenty years in English language and focused on epidemiology, risk factors, consequences, prevention and treatment of PPD especially in Pakistan.

Results: Postpartum depression is a prevalent illness, with complications that are severe. The causes can be situational or maternal and thus, recommended prevention is to reduce the burden related to public health. If primary prevention fails, programs for screening to detect early can be well thought-out for timely treatment. If there is any progress in the disease, implementation of antidepressant, effectual psychotherapy besides the recommended diet are beneficial. Exercise and appropriate sleep as well are also recommended for the patients. Moreover, barriers to each level of prevention exist due to maternal factors, healthcare factors and socioeconomic factors in Pakistan.

Conclusion: Results suggest that women do not proactively seek help when suffering from postpartum depression due to many factors, the root cause of which is lack of awareness especially in developing countries like Pakistan. Since this ignored illness can lead to serious complications, the issue should be addressed to promote public health.

Keywords: Postpartum depression, maternal health, public health, socioeconomic barriers, epidemiology, literature review.

doi.org/10.21089/njhs.21.0024

\section{INTRODUCTION}

Motherhood is the most rewarding experience in the life of a woman. Birth of a baby entails new aspirations and hopes. But what if this blessing of a baby is bringing weepiness and anxiety instead of excitement and delight? And if in some conditions the mother considers killing her baby or herself instead of feeling privileged on the greatest gift of God? All this turns to reality when a woman is suffering from Postpartum Depression (PPD). PPD, a term used for depression that prevails in the postpartum period, therefore, in clinical practice and research is reviewed till one year after the child's birth [1]. Accordingly, in Diagnostic and Statistical Manual5 , the onset of PPD occurs within four weeks of child birth [2]. It usually effects on $10-15 \%$ women and is considered as

\footnotetext{
*Address correspondence to this author at the Aga Khan University Hospital, Karachi, Pakistan.

E-mail: rfa.alini@gmail.com
}

a frequent complication in pregnancy [3]. In Asian countries the prevalence rate ranges from $3.5 \%$ to $63.3 \%$ [4]. Pakistan has the highest prevalence amongst Asian countries that is $28 \%-63 \%$, [5]. Researches on prevalence of PPD conclude developing countries face its greatest burden [6]; in fact, the prevalence rate of developing countries ranges from being equal to double from that of the developed world [7]. More than half of the cases in Pakistan go unrecognized [8]. The consequences of PPD go beyond the mother and affect the husband and the child as well and can even lead to infanticide or maternal death, often by suicide. Despite several treatment options, not all women are assessed for PPD and receive its treatment [9]. Therefore, highlighting the epidemiology of PPD is very important in public health.

The history of PPD shows that, this ailment was named in 1850. Record of PPD dates back from the time of Hippocrates; however, its symptoms remained unidentified for centuries. The history of PPD is grey as nobody was ever able to 
recognize it. Some women were not able to verbalize their symptoms because of shame and guilt; others were treated by spiritual healers. Rare cases were reported to healthcare departments and those were treated with unusual treatment. Finally, following higher incidence rate in the nineteenth century, it was added into medical glossary as "Postpartum depression" [10].

\section{MATERIALS AND METHODS}

Comprehensive review of literature was done from various articles using SAGE, PubMed, Cochrane, CINAHL, Medline, ASSIA, WHO Reproductive Health Psychology, PsychInfo, using search terms such as Postpartum Depression, postnatal depression, perinatal depression, Pakistan, Asia, public health, epidemiology, husband, child, detection, culture, barriers etcetera. Moreover, links to "related articles" were accessed in electronic databases. Inclusion criteria included articles in English as funds were not available to translate articles published in non-English languages. Articles published since the last ten years were preferred; however, few older articles were also included as some key studies conducted many years ago have not been superseded in recent years. Research studies were selected that included incidence, prevalence, risk factors of PPD especially in Pakistan. Furthermore, prevention and treatment options for PPD were also examined.

\section{RESULTS}

Findings of the studies suggested many factors to be involved with PPD. According to the Wheel Model of Disease Causation, environmental factors and host factors cause a disease. The wheel model for PPD as adapted from the work of Howell, Mora, Horwitz and Leventhal, 2005 as cited in Callister, Beckstrand and Corbett (2010) represents maternal characteristics (host) and situational demands (environment) to cause PPD [11] (Appendix A).

Integrating the natural history of PPD (Appendix B), the susceptibility stage is illustrated by the model which indicates factors which makes person susceptible to PPD. Categories including maternal characteristics and situational demands, leading to either a positive maternal role or PPD. Successful coping results in the attainment role of mother and mother-infant healthy attachments are important. Conversely, the failure will result in PPD. Some major causes of PPD are discussed below.

Different sociocultural practices include seclusion and number of dietary limitations during the postpartum period in many cultures and a mixture of other cultural taboos are also few factors that are indirectly or directly associated [11]. One more factor which causes many disturbing thoughts to mother regarding upbringing of the baby is poverty $[12,13]$. People living in poverty are less likely to have access to satisfactory housing, healthy nutrition, clean water, and appro- priate health services. Childbirth and caring for a baby in addition to pre-existing responsibilities can aggravate the stress level [14]. Furthermore, lack of husband attachment and family support are augmenting factors for PPD, as women during postpartum period need emotional attention and quality care, therefore, its lacking can result in PPD [15]. According to a research conducted in Pakistan, when father and grandmothers were involved in taking care of the babies, the mothers had time to rest, thereby, decreasing the depressive symptoms [16]. Lastly, PPD occurrence is linked with different types of personality. Women that are introvert are much inclined towards PPD because they try to hide their feelings and PPD can easily be provoked in this type of nonventilation of feelings [17].

At sub-clinical stage, several hormonal changes take place during pregnancy, for instance, melatonin and cortisol imbalance disturbs sleep cycle [18] that progresses to a common clinical stage when mothers exhibit symptoms including mood swings, anxiety, insomnia, decreased concentration and lack of joy, pleasure and interest in life [19]. The last stage is convalescent stage in which the mother can recover by abiding tertiary preventions discussed later in this paper. However, if ignored, the consequences may result in chronic depression, suicide and homicide [1].

\section{LEVELS OF PREVENTION}

To overcome and decrease the disease load and its amplification of symptoms, following prevention approaches are suggested. Primary prevention is intended to prevent a disease or condition from occurring in the first place. For primary prevention, improvements in one or more significant risk factors could reduce PPD. Community teachings to all women of reproductive age, whether in universities or different areas of workplace, can prevent its incidence. Likewise, risk assessment and clinical analysis in earlier stages or during preconception in pregnancy are practical strategies to identify PPD early because it helps in identifying history of the depressive episodes, premenstrual dysphoric symptoms, family history, role changes, life stressors, concern to patient's mood swings and different attitudes in pregnancy [18]. However, if primary prevention fails, secondary prevention can help avert PPD. At secondary level, screening tools are used for early identification of disease. Two most common screening tools used for PPD are Patient Health Questionnarre-9 and Edinburgh Postnatal Depression Scale [20]. These tools assess clients in several dimensions which include sleeping/eating disturbances, cognitive impairment, anxiety/insecurity, emotional liability, shame/ guilt, loss of self, and contemplating harming oneself. These screening tools can also be used in gynaecology and obstetrics clinics by healthcare professionals to identify high risk mothers before disease affects them [21]. However, because of the limited use of such tools in Pakistan, many sufferers end up with PPD [22]. Consequently, tertiary prevention is commonly 
used in Pakistan because of the negligence of primary and secondary prevention. Tertiary prevention necessitates large chunk of interventions which include antidepressants, cognitive-behavioural therapy, social support, interpersonal psychotherapy, dietary management, sleep, exercise, stress reduction, breastfeeding and family planning. Depending on individual's resilience, the intervention is decided from aforementioned. [18].

Firstly, antidepressants like serotonin-selective reuptake inhibitors (SSRI) are frequently used to decrease major depressive symptoms and to reduce relapse of PPD [23]. However, use of antidepressants in breastfeeding women is controversial as they pass into breast milk, thus delivered to the infant. Yet, the quantity of drug found in infants' sera found to be minute with no evidence of infant's harm due to mother's medication usage [1]. At any case, National Institute for Clinical Excellence (NICE) and the English Department of Health have recommended many psychological therapies. Of these psychological measures, Cognitive Behavioural Therapy (CBT) has found to be as effective as antidepressants in treating severe PPD [9]. CBT decreases emotional transition for mothers who struggle emotionally with pregnancy and role changes. Psychotherapy and counselling sessions help reduce emotional instability and construct relaxation techniques. Health professionals can facilitate clients in mindfulness exercises and relaxation techniques to decrease emotional distress and embrace positivity toward role transitions [18].

Referring to psychotherapy, sessions can be conducted for couples to explain the partners about role transition that occurs during motherhood. This can help develop social support for mothers by their partners [18]. Thirdly, proper nutrition is beneficial in all levels of prevention. Vitamin D and vitamin B3 increases the risk of depression, hence, should be avoided in mothers with greater risk of depressive symptoms. Moreover, n-3 Essential fatty acids are important in preventing depression. Also, Methylfolate is effective to reduce depressive symptoms. [18].

Several studies imply that aerobic exercise protects neurons from the toxic effects of depression. Physical activity for at least thirty minutes can be used as primary prevention for PPD. Nevertheless, if disease develops, postnatal exercises can manage the symptoms [18].

In addition, perinatal alterations in hormonal circadian rhythms of cortisol and melatonin linked to sleep-wake cycle play an important role in depressive symptoms. A study showed improved hormonal balance in women with PPD when time was scheduled to meet and feed the baby and when there were minimal doctors' and nurses' interruptions in private wards. These strategies showed effectiveness for decreasing depressive symptoms [24].
Furthermore, breastfeeding reduces depressive symptoms by decreasing cortisol level. Conversely, in some studies, breastfeeding stimulates depressive symptoms due to disturbances in sleep-wake cycle, problems in milk production and physical wellbeing of child (decrease sucking). For that purpose, counseling on breastfeeding before postpartum period can decrease stressors [18].

Several studies also indicate increased depressive symptoms due to unintended pregnancies. Counseling sessions for couples to guide them related to the efficacy of family planning method can reduce depressive symptoms. Moreover, women with PPD can use a reliable family planning method to reduce its relapse [18].

Findings of a current study in Pakistan also reveal that mothers of preterm neonates are at a high risk for developing PPD. Also, Pakistani mothers who experience preterm birth for a second time have a higher chance to develop PPD [25]. Early interventions can be planned that focus on educating parents regarding preterm neonates' behavioral cues and feeding readiness, in addition to nutritional requirements to promote growth and development. Sufficient knowledge regarding upbringing of a preterm may decrease depressive symptoms [26].

\section{DISCUSSION}

Despite these measures, there exist various barriers for each level of prevention. Firstly, many women assume that their symptoms are usual part of motherhood and the consequence of fatigue, personal weakness or relationship difficulties rather than depression. These perceptions when supported by family interfere with timely support [27]. Thus, healthcare professionals who deal with perinatal women should guide them regarding manifestations of PPD before its risk period, highlighting the myths and misconceptions to the patient and her family and reassure social support for role adjustment. Some studies also indicate that less educated and nonEnglish speaking women are less aware of PPD. Therefore, keeping demographic profiles of women in mind is significant. Also, different forms of public education can be used including face to-face communication, internet, television, and newsprint. Healthcare professionals should ensure that all information on PPD is culturally sensitive and correct, and organization providing inaccurate information should be approached for clarification. All information on printed or electronic media regarding PPD must be verified for content accuracy and it should be ensured that the literacy level does not exceed Grade five reading level [27].

It has also been found via several studies that although women are aware of their condition, utilization of mental health services during postpartum period is minimal [28]. In a huge community screening study of PPD, only $3.4-6 \%$ women screening positive opted for pharmacological treatment at 
three and four months postpartum and only $12 \%$ women received psychotherapy [29]. A national survey on the symptoms of Postpartum Depression concluded that $57 \%$ of mothers exhibiting fair to severe symptoms are unable to seek healthcare assistance [4] General barriers especially in Pakistan include feelings of failure, shame, lack of awareness about treatment options and place to seek treatment, childcare and family responsibilities, stigma, doubt about treatment efficiency and perceived inadequate time. Thus, home visits to pregnant and postpartum women should be conducted by community health workers to provide them education, social support, access to benefits and referral to mental health and healthcare delivery system after essential survey and screening. To do so, the first step is the training of community health workers themselves. Training of community workers is important because it is found to be difficult for them to distinguish between PPD and normal reaction towards stressful life event. However, it was also observed that people are reluctant to allow community health workers to enter their homes because of some cultural taboos and past experiences. At times, when people allow community health workers in homes, they are not willing to talk about mental health issues and are reluctant that these issues are not as much important and must not be talked about and should be concealed, otherwise, family name will be spoiled or people will talk about it. [30] At other instances, head of the family influences the responses to the questions that are asked by community health nurses to clients, which hinders the screening process. Luckily, if the issues are discussed, the EPDS screening tool has been found to both overidentify and under-identify cases. Nevertheless, after the screening process, several barriers to referring postpartum depressive mothers to mental health services have been identified. These include practical barriers (logistical impediments like conveyance issues, inability to afford health care, sometimes childcare barrier and health insurance limitations like difficulty paying for the daycare as children are not permitted to be taken at sessions), personal barriers (lack of motivation, mistrust, fear of mental health service systems and low priority for mental health related to other major stressors in life), mental health system barriers (lack of services, in-hospitable conduct of front line mental health staff), internal agency barriers (limited services, scarce resources and overburdened staff), and stigma of being labeled as having psychiatric disorder.

Many of these barriers can be addressed. Firstly, initial mental health appointment can be arranged to diagnose mental health issues along with transportation service and patients can be accompanied to mental health services by community health workers [31]. Secondly, community health facilities can collaborate with mental health agencies to ensure delivery of appropriate services. Thirdly, healthy clients who recovered from the treatment regime can be invited to share their inspirational stories to motivate patients to opt for treatment positively. Above all, building trust and making associations is a key which can be achieved by offering general support and reducing client's isolation [32]. Support can be provided by normalizing symptoms and situations by explaining the way to interpret their feelings, thoughts and behaviors and assisting them to understand their symptoms. For uninsured women, referral for first appointment should be offered to an on-site social worker without fee. However, research trials are required to evaluate the effectiveness of the recommendations and should be planned and designed to conquer frequent barriers in community settings. It is so far an important tool in research and treatment with correct diagnosis [1].

Despite aforementioned solutions and limitations, epidemiological studies on PPD are limited. Most researchers have used convenience sampling. Some studies have a broader reach but none have been designed to determine incidence, prevalence and onset relative to childbirth. While good work has been done to identify consequences of maternal depression on child, however, studies on its longstanding effects to the child and evidence of benefits of effective PPD treatment on child are relatively limited. Moreover, evaluation of interventions to specifically address the parenting of postpartum depressed mothers is required [33]. Furthermore, it is likely that PPD reflect a specific childbirth related diathesis which may represent a specific sensitivity to disruption in ovarian hormones occurring in menstruation, pregnancy, childbirth, and perimenopause. If this diathesis prevails, determining its nature is a significant goal for research in order to develop specific intervention for prevention and treatment PPD in women who present with this diathesis. Besides, more research needs to be done on the screening process for PPD to establish its outcome on women's general functioning and resulting healthcare delivery. Also, cultural differences and demographic variables should be well thought-out in the upcoming researches. More research is needed to establish the understanding of public regarding self-care strategies which include proper nutrition, adequate sleep, exercise, emotional support, and pursuing professional assistance when required.

\section{CONCLUSION}

In conclusion, PPD is a prevalent illness in Pakistan which can lead to severe complications. The causes of PPD can be maternal or situational, thus, its prevention is recommended to reduce its burden in public health. If primary prevention fails, screening for timely detection should be done, however, if the diseases progresses, antidepressant compliance and effective psychotherapy are beneficial along with recommended nutrition, adequate sleep and proper exercise. Yet, more research is required to uncover its several important aspects. After all, mother-child bond is the most beautiful 
bond in the world which should not be ruined by postpartum depression.

\section{FUNDS FOR STUDY}

As the manuscript type of this article is literature review, no funds were required to undertake this endeavor.

\section{IRB/IEC Approval}

As the article requested for publication is literature review, it is not required to obtain approval from Institutional Review Board (IRB) or Institutional Ethics Committee (IEC).

\section{ACKNOWLEDGEMENT}

Declared none.

\section{CONFLICT OF INTEREST}

Declared none.

\section{SOURCE}

Centers for Disease Control and Prevention. Principles of epidemiology, 2nd ed. Atlanta: U.S. Department of Health and Human Services; 1992.

\section{APPENDIXES}

\section{Appendix A: Wheel model for PPD}

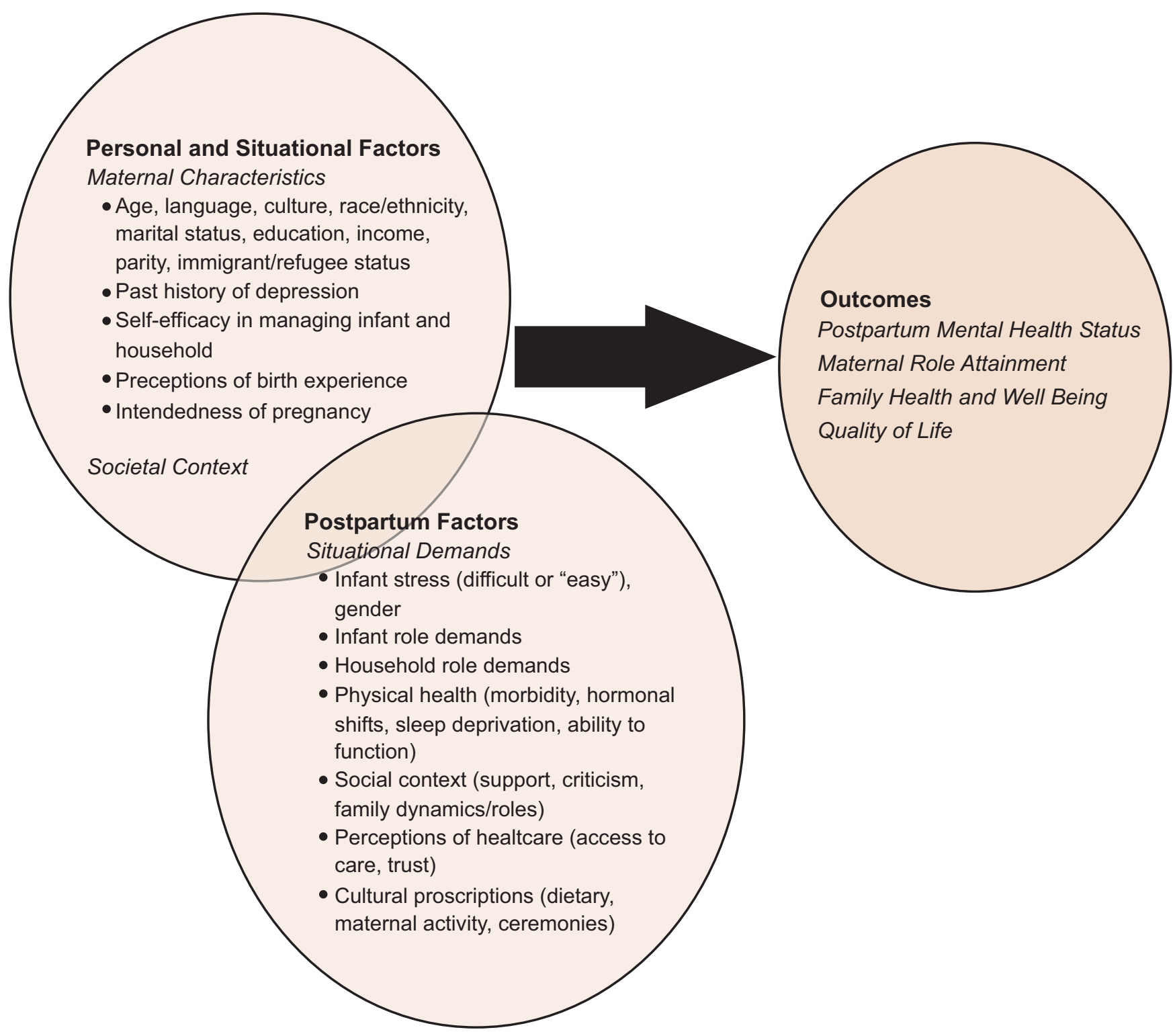




\section{Appendix B: Natural History of Disease}

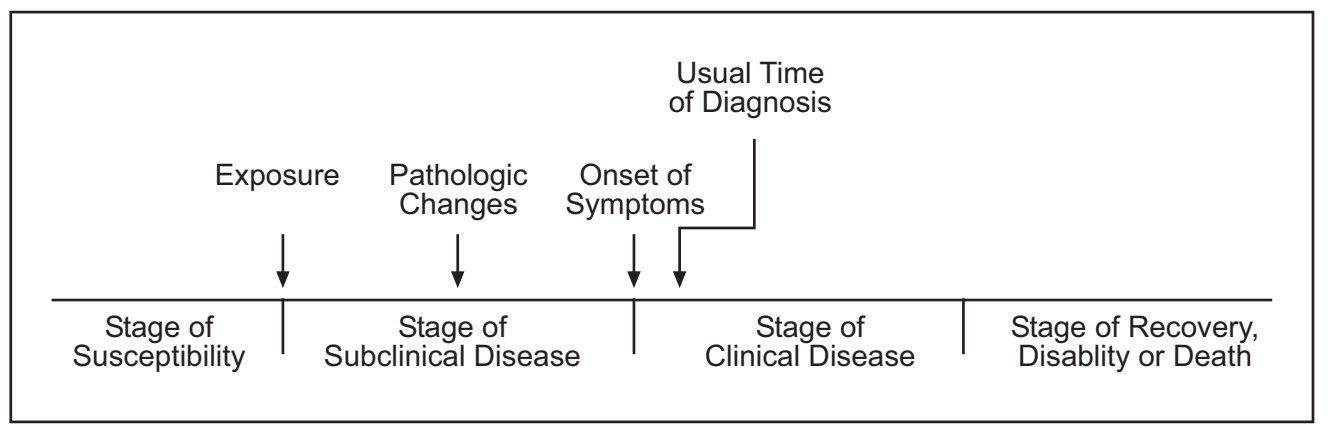

\section{REFERENCES}

[1] O' Hara M. Postpartum depression: what we know. Journal of Clinical Psychology. 2009; 65(12): 1258-1269.

[2] DSM-5 American Psychiatric Association. Diagnostic and statistical manual of mental disorders. Arlington: American Psychiatric Publishing. 2013.

[3] Vigod S, Villegas L, Dennis C, Ross L. Prevalence and risk factors for postpartum depression among women with preterm and lowbirth-weight infants: a systematic review. BJOG: Inter. J. Obs. Gynaecol., 2010; 117(5): 540-550.

[4] Klainin P, Arthur D. Postpartum depression in Asian cultures: A literature review. Inter. J. Nursing Studies. 2009; 46(10): 13551373.

[5] Gulamani S, Shaikh K, Chagani J. Postpartum Depression in Pakistan. Nursing for Women's Health. 2013; 17(2): 147-152.

[6] Mayberry L, Horowitz J, Declercq E. Depression Symptom Prevalence and Demographic Risk Factors Among U.S. Women During the First 2 Years Postpartum. J. Obs. Gynecol. Neonat. Nursing., 2007;36(6):542-549.

[7] Husain N, Bevc I, Husain M, Chaudhry IB, Atif N, Rahman A. Prevalence and social correlates of postnatal depression in a low income country. Archives of Women's Mental Health. 2006; 9(4): 197-202.

[8] Emmanuel A, Mazhar S, Shahid A. Predictors of postpartum depression among Pakistani women delivering in tertiary care hospital. J. Soc. Obs. Gynecol. Pak., 2016; 1(1): 33-40.

[9] Almond P. Postnatal depression: A global public health perspective. Perspectives in Public Health. 2009; 129(5): 221-227.

[10] Wolf.R, Post-partum depression: A history. 2010. Retrieved from http://www.healthguideinfo.com/postpartum- depression/p99788/

[11] Callister L, Beckstrand R, Corbett C. Postpartum Depression and Culture. MCN, Am. J. Maternal/Child Nur., 2010;35(5):254-261

[12] Muñoz R. Prevention of Postpartum Depression in Low-Income Women: Development of the Mamás y Bebés/Mothers and Babies Course (Muñoz et al.). Cognitive and Behavioral Practice. 2007; 14(1): 121.

[13] Weiss BD, Sheehan CP, Gushwa LL. Is low literacy a risk factor for symptoms of depression in postpartum women? J. Reprod. Med., 2009; 54(9): 563-8.

[14] Coast E, Leone T, Hirose A, Jones E. Poverty and postnatal depression: a systematic mapping of the evidence from low and lower middle income countries. Health \& Place, 2012; 18(5): 11881197.

[15] Dolbier C, Rush T, Sahadeo L, Shaffer M, Thorp J. Relationships of Race and Socioeconomic Status to Postpartum Depressive Symptoms in Rural African American and Non-Hispanic White Women. Mat. Child Health J., 2012; 17(7): 1277-1287.
[16] Khanam M, Sultana A, Siddiqui SH, Rehman K. Screening for postpartum depression in recently delivered mothers. Pak. J. Med. Sci., 2011; 27 (2): 320-324.

[17] Tian T, Li Y, Xie D, Shen Y, Ren J, Wu W et al. Clinical features and risk factors for post-partum depression in a large cohort of Chinese women with recurrent major depressive disorder. J. Affect. Dis., 2012;136(3):983-987.

[18] Miller LaRusso E. Preventing Postpartum Depression. Psychiatric Clinics of North America. 2011;34(1):53-65.

[19] Friedman S, Resnick P. Postpartum depression: an update. Women's Health. 2009; 5(3): 287-295.

[20] Zubaran C, Schumacher M, Roxo M, Foresti K. Screening tools for postpartum depression: validity and cultural dimensions. African Journal of Psychiatry. 2011; 13(5)

[21] Hegadoren K, Norris C, Lasiuk G, Silva D, Chivers-Wilson K. The many faces of depression in primary care. Textocontexto - enferm. 2009; 18(1): 155-164.

[22] Rahman A, Iqbal Z, Lovel H, Shah MA. Screening for postnatal depression in the developing world: A comparison of the WHO self-reporting questionnaire (SRQ-20) and the Edinburgh postnatal depression screen (EPDS). J. Pak. Psy. Soc., 2005; 2: 69-72.

[23] Gill D, Hatcher S. Antidepressants for depression in medical illness. The Cochrane Library. 2000 Oct.

[24] Yim I, Glynn L, DunkelSchetter C, Hobel C, Chicz-DeMet A, Sandman C. Risk of Postpartum Depressive Symptoms With Elevated Corticotropin-Releasing Hormone in Human Pregnancy. Archives of General Psychiatry. 2009; 66(2): 162

[25] Gulamani SS, Premji SS, Azam SI, Kanji Z. Preterm birth a risk factor for postpartum depression in Pakistani women. Open J. Dep., 2013; 2(04): 72.

[26] Maguire CM, Bruil J, Wit JM, Walther FJ. Reading preterm infants' behavioral cues: An intervention study with parents of premature infants born< 32 weeks. Early human development. 2007; 83(7): 419-24.

[27] Sealy P, Fraser J, Simpson J, Evans M, Hartford A. Community Awareness of Postpartum Depression. J. Obs. Gynecol. Neonat. Nursing., 2009; 38(2): 121-133.121-133.

[28] Boyd R, Mogul M, Newman D, Coyne J. Screening and Referral for Postpartum Depression among Low-Income Women: A Qualitative Perspective from Community Health Workers. Depression Research and Treatment. 2011; 2011: 1-7.

[29] Horowitz JA, Cousins A. Postpartum depression treatment rates for at-risk women. Nursing research. 2006; 55(2): S23-7.

[30] Ng C. The stigma of mental illness in Asian cultures. Aust NZ J Psy., 1997; 31(3): 382-390. 
[31] Dennis Chung-Lee L. Postpartum Depression Help-Seeking Barriers and Maternal Treatment Preferences: A Qualitative Systematic Review. Birth. 2006; 33(4): 323-331.

[32] Duran L. Motivating Health: Strategies for the Nurse Practitioner. J. Am. Acad. Nurse Pract., 2003;15(5):200-205.
[33] Shaw E, Levitt C, Wong S, Kaczorowski J. Systematic Review of the Literature on Postpartum Care: Effectiveness of Postpartum Support to Improve Maternal Parenting, Mental Health, Quality of Life, and Physical Health. Birth. 2006; 33(3): 210-220.

(C) 2017 National journal of health sciences.

This is an open-access article. 